\title{
Links of plane curve singularities are $L$-space links
}

\author{
EUGENE GORSKY \\ ANDRÁS NÉMETHI
}

\begin{abstract}
We prove that a sufficiently large surgery on any algebraic link is an $L$-space. For torus links we give a complete classification of integer surgery torus links we give a complete classification of integer surgery coefficients providing $L$-spaces.
\end{abstract}

14H20, 57M27

Definition 1 A 3 -manifold $Y$ is called an $L$-space if it is a rational homology sphere and its Heegaard-Floer homology has minimal possible rank: rank $\widehat{\mathrm{HF}}(Y)=$ $\left|H_{1}(Y, \mathbb{Z})\right|$.

We refer the reader to Ozsváth and Szabó $[17 ; 20 ; 18]$ for definitions of Heegaard-Floer homology, and to Hedden [8] and Ozsváth and Szabó [19] for a detailed discussion of the properties of $L$-spaces. Let $K \subset S^{3}$ be the embedded link of a complex plane curve singularity with $r$ components $K=K_{1} \cup \cdots \cup K_{r}$. The following theorem is the main result of this note. We use it in our work [3] to compute the Heegaard-Floer homology for algebraic links.

Theorem 2 Every algebraic link $K \subset S^{3}$ is an $L$-space link. This means that an integral surgery of $S^{3}$ along the link components $K_{i}$ with all coefficients sufficiently large provides an $L$-space.

For $r=1$, the result was proven by Hedden [8, Theorem 1.10]. Our proof is of a different nature, is extremely short, and provides the argument uniformly for any $r$. It is based on some facts from the theory of normal surface singularities and plumbed 3-manifolds. For plumbing calculus, which modifies the possible graph representations of the same 3-manifold, see Neumann [15]. We will need the following facts:

(a) A connected negative-definite plumbing graph is called a smooth graph if by consecutively blowing down $(-1)$-vertices, we can blow down the graph to the empty graph. Such a graph represents $S^{3}$. When we resolve plane curve singularities and blow up $\left(\mathbb{C}^{2}, 0\right)$ in several infinitely near points, we obtain such a graph.

(b) A surface singularity is rational if its geometric genus is 0 . This property can be verified at the level of its negative-definite plumbing graph (such a graph is called a rational graph); see Artin [1; 2] and Laufer [11]. 

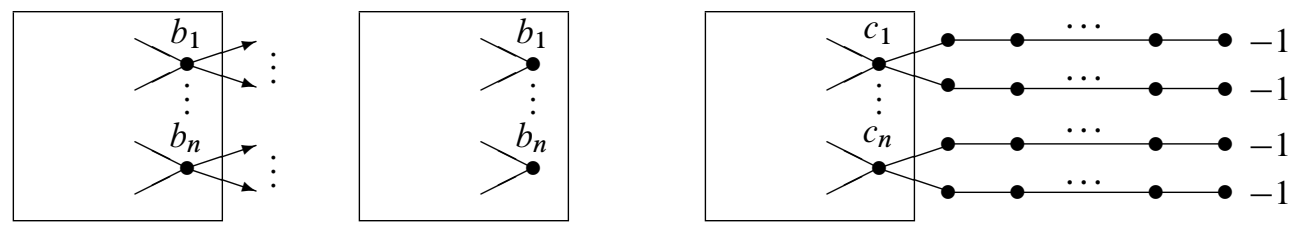

Figure 1: The graphs $\Gamma_{K}, \Gamma_{0}$ and $\widetilde{\Gamma}_{0}$

If we blow up a rational graph, we in turn obtain a rational graph. Any subgraph of a rational graph is rational; see eg Laufer's criterion [11]. Since smooth graphs are rational, subgraphs of smooth graphs are rational (these are called sandwiched graphs; see Spivakovsky [21]). The following remark describes two useful consequences of Laufer's criterion.

Remark 3 (cf Spivakovsky [21, Remark 2.3, Proposition 2.4]) For each vertex $v$ of the negative-definite plumbing graph $\Gamma$, define $w(v)=-\left(E_{v}, E_{v}\right)$, where $E_{v}$ is the corresponding curve. Let $\gamma(v)$ denote the valency of $v$ in $\Gamma$.

(a) If $\Gamma$ is rational, then $w(v) \geq \gamma(v)-1$ for all $v$.

(b) If $w(v) \geq \gamma(v)$ for all $v$, then $\Gamma$ is rational (and, in fact, sandwiched). Such graphs are called minimal rational.

A key ingredient in the proof of Theorem 2 is the following result.

Theorem 4 (Némethi [14, Theorems 6.3, 8.3]) A 3-manifold plumbed from a rational graph is an $L$-space.

Let $C=C_{1} \cup \cdots \cup C_{r} \subset \mathbb{C}^{2}$ be the plane curve singularity corresponding to $K$, such that the link of the irreducible component $C_{i}$ is the knot $K_{i} \subset S^{3}$. The possible minimal embedded resolution (plumbing) graphs of complex plane curve singularities are well known. We will represent such a graph in a schematic way by the graph $\Gamma_{K}$ shown in Figure 1, emphasizing only those exceptional curves, say $E_{1}, \ldots, E_{n}$, which intersect one or more strict transform components. The strict transform components are encoded by arrowheads. The number of arrowheads supported by $E_{i}$ is $a_{i}$, and the self-intersection number of $E_{i}$ is $b_{i}(1 \leq i \leq n)$. Definitely, some of $b_{i}$ 's are -1 .

If we delete the arrowheads, we get a smooth graph $\Gamma_{0}$ (the second graph in Figure 1). If we blow up $\Gamma_{0}$ a few times (starting with $E_{i}$ 's and continuing with the newly created $(-1)$-vertices), we obtain another graph $\widetilde{\Gamma}_{0}$, which is again smooth. This is the third graph in Figure 1. The unmarked vertices are $(-2)-$ vertices, and $c_{i}=b_{i}-a_{i}$. The length of the newly created legs can be different: the number of $(-2)$-vertices in the $(i, j)^{\text {th }}$ leg is $\left(k_{i j}-1\right)$, where $k_{i j} \geq 1$ for all $1 \leq i \leq n, 1 \leq j \leq a_{i}$. 


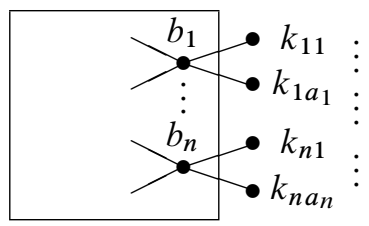

Figure 2: The graph $\Gamma$, where $k_{i j} \geq 1$ for all $1 \leq i \leq n, 1 \leq j \leq a_{i}$

Lemma 5 For $k_{i j} \geq 1$, the 3-manifold represented by the graph $\Gamma$ shown in Figure 2 is an $L$-space.

Proof By plumbing calculus (blowing up several times the right-most edges), $\Gamma$ is equivalent to the graph $\Gamma^{\prime}$ obtained from the smooth graph $\widetilde{\Gamma}_{0}$ by deleting the $(-1)-$ vertices from the end of the new legs. Hence $\Gamma^{\prime}$ is a subgraph of a smooth graph, thus it is rational. In particular, it represents an $L$-space.

Proof of Theorem 2 Suppose that the plane curve singularity corresponding to $K$ is given by the equation $\left\{\prod_{i, j} f_{i j}(x, y)=0\right\}$. Consider a positive surgery on $S^{3}$ along the link $K$, where the surgery coefficient for the component $K_{i j}$ equals $d_{i j}$. It is known that $S_{\left\{d_{i j}\right\}_{i j}}^{3}(K)$ is a plumbed 3-manifold with the plumbing graph $\Gamma$, such that the parameters $k_{i j}$ are defined by the equation $k_{i j}=d_{i j}-m_{i}$, where $m_{i}$ are the multiplicities of the pullback of $f_{i j}$ on the divisor $E_{i}$. For $d_{i j}>m_{i}$ the surgery space is an $L$-space by Lemma 5 .

Remark 6 (a) The above bound $k_{i j} \geq 1$ is not optimal, usually one can find a collection of numbers $B_{i j}$ less than 1 , such that all the surgeries with coefficients $k_{i j} \geq B_{i j}$ provide $L$-spaces. But for the coefficients $B_{i j}=1$ the proof is extremely transparent (and, for smaller coefficients, singularity theory is harder to apply).

(b) If $a_{i}=1$ for all $i$, then one can take $k_{i 1} \geq 0$, and the surgery manifold is still an $L$-space. For example, if we take all of them equal to zero, then $\Gamma$ is equivalent to a graph obtained from $\Gamma$ by deleting the 0 -vertices and the supporting $E_{i}$-vertices. This graph is not connected, but each component is a subgraph of a smooth graph. Hence the corresponding 3-manifold is a connected sum of $L$-spaces, which is again an $L$-space. The proof of the general case $k_{i 1} \geq 0$ (with $a_{i}=1$ ) is a combination of this argument and the proof of Theorem 2.

(c) It is known that all algebraic knots (with one component) can be presented as iterated cables of the trivial knot. Hedden [8] proved that the $p q$ surgery of $S^{3}$ along an algebraic knot is an $L$-space, where $p$ and $q$ are the parameters of the last cabling. One can check that $p q=m_{1}$ in this case. See also Hom [9] for a complete description of $L$-space surgeries of cable knots. 


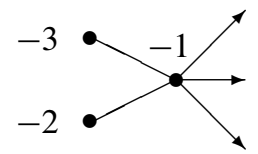

Figure 3: Plumbing graph for the singularity $x^{6}+y^{9}=0$

It turns out that the set of all surgeries on a link providing an $L$-space has an interesting structure. This is a new phenomenon compared with the irreducible case, where (eg by Hom [9]) a $d$-surgery on a nontrivial $L$-space knot $K$ provides an $L$-space if and only if $d \geq 2 g(K)-1$, and hence $d$ runs in a half line. The following theorem provides a description of $L$-space surgery coefficients for torus links.

Theorem 7 Let $p, q>1$ be two coprime integers, $r \geq 1$ and $\left(d_{1}, \ldots, d_{r}\right) \in \mathbb{Z}^{r}$. Assume that $d_{i} \neq p q \pm 1$ for all $i$. Then $\left(d_{1}, \ldots, d_{r}\right)$-surgery on $(p r, q r)$-torus link is an $L$-space if and only if the surgery space is a rational homology sphere and one of the following conditions hold:

(a) $d_{i}=p q$ for some $i$,

(b) $d_{i}>p q+1$ for all $i$,

(c) $d_{i}<p q-1$ for all $i$ and $\max \left(d_{i}\right) \geq p q-p-q$.

Remark 8 It is easy to see that $d_{i}=d_{j}=p q$ for $i \neq j$ yields infinite $H_{1}$ for the surgery space, so Theorem 7(a) can only be true for exactly one $i$.

Remark 9 The surgeries with $d_{i}=p q \pm 1$ can also be studied by a (rather long) case by case analysis. In particular, consider the following cases (not an exhaustive list):

(a) The $(p q \pm 1, d)$-surgery on $(2 p, 2 q)$-torus link is an $L$-space for all $d$.

(b) The $\left(p q+1, p q-1, d_{3}, \ldots, d_{r}\right)$-surgery on $(p r, q r)$-torus link coincides with $\left(d_{3}, \ldots, d_{r}\right)$ surgery on $(p(r-2), q(r-2))$-torus link. In particular, $(p q+1, p q-1, d)$-surgery on a $(3 p, 3 q)$-torus link is an $L$-space if and only if $d \geq p q-p-q$.

Proof The plumbing graph of the surgery space can be described as above, with $k_{i}=d_{i}-p q$; see Figure 3 for the case of the $(6,9)$-torus link with three components, and also Kadokami and Shimozawa [10] for more details. It is star-shaped, so the surgery space $Y:=S_{d}^{3}(L)$ is Seifert fibered. By Neumann [16], the intersection matrix for the Neumann normal form is negative-definite either for $Y$ or for $-Y$. Any negative-definite Seifert graph is almost rational in the sense of Némethi [14], so it represents an $L$-space if and only if it is rational.

If $d_{i}=p q$ then $k_{i}=0$ and the surgery space is a connected sum of lens spaces. From now on we will assume that $k_{i} \notin\{-1,0,1\}$ for all $i$. 
If $k_{i}>1$ for all $i$, then we get an $L$-space by Lemma 5. Suppose that the $k_{i}$ have different signs, for example $k_{1}>0$ and $k_{2}<0$. To obtain the normal form of $Y$, one must blow up the vertices with positive $k_{i}$, of which there are at most $r-1$, so the self-intersection of the central vertex in the normal form is greater than or equal to $-1-(r-1)=-r$, while its valency is $r+2$. To obtain the normal form of $-Y$, one reverses all signs and blows up all positive vertices (at most $r+1$ of them), so the self-intersection of the central vertex is greater than or equal to $1-(r+1)=-r$. If $Y$ is an $L$-space, either $Y$ or $-Y$ should be a negative-definite rational graph, which contradicts Remark 3(a).

Finally, suppose that $k_{i}<0$ for all $i$. In this case we can use a theorem of Lisca and Stipsicz [12] describing Seifert fibered $L$-spaces. Suppose that

$$
1 \geq \alpha_{1} \geq \alpha_{2} \geq \cdots \geq \alpha_{r+2} \geq 0
$$

are the Seifert invariants of singular fibers, and the central vertex has self-intersection $(-1)$. Then the corresponding 3-manifold is an $L$-space if and only if there are no coprime integers $(l, m)$ such that

$$
m \alpha_{1}<l<m\left(1-\alpha_{2}\right) \text { and } m \alpha_{i}<1 \text { for } i \geq 3 .
$$

One can check that in our situation $\alpha_{1}$ and $1-\alpha_{2}$ are two neighboring fractions in the sense of Farey series (ie $\alpha_{1}=a / p$ and $1-\alpha_{2}=b / q$ with $a q-b p= \pm 1$ ), and $\alpha_{i}=1 /\left|k_{i-2}\right|$ for $i \geq 3$.

It is well known (see eg Graham, Knuth and Patashnik [5, Section 4.5]) that the fraction $(a+b) /(p+q)$ has the least possible denominator among rational numbers between $a / p$ and $b / q$. Therefore if $k_{i} \geq-p-q$ for some $i$, then (1) cannot be satisfied and $Y$ is an $L$-space; if $k_{i}<-p-q$ for all $i$, then $(l, m)=(a+b, p+q)$ satisfies (1) and $Y$ is not an $L$-space.

Example 10 For $r=1$ the conditions of Theorem 7 are equivalent to the inequality $d_{1} \geq p q-p-q=2 g(T(p, q))-1$, which also follows from Hom [9].

Example 11 Consider the case $r=2$. The set of all pairs $\left(d_{1}, d_{2}\right)$ providing an $L$-space is shown in grey in Figure 4. The point $(p q, p q)$ (marked by a star) provides a surgery with infinite first homology and should be excluded. See also Liu [13, Proposition 6.3 and Figure 6.1] for the set of $L$-space surgeries on $(2,2 n)$ torus links and our previous work [4] for a discussion of this set for more general $L$-space links.

Theorem 2 can be reinterpreted as follows: start with a negative definite nonminimal plumbing graph $\Gamma_{0}$ of $S^{3}$, put some arrows on the vertices, and regard them as link components of $K$ in $S^{3}$. Then the surgery manifold with all sufficiently large surgery coefficients is an $L$-space. 


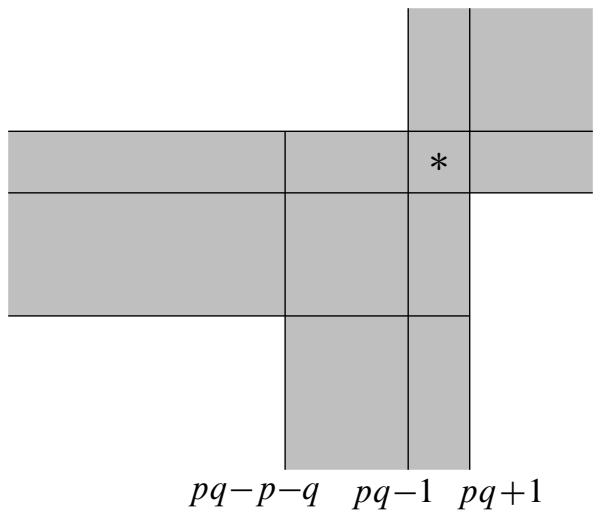

Figure 4: Possible $L$-space surgery coefficients for $(2 p, 2 q)$ torus link, where $*$ marks $(p q, p q)$

The proof works entirely unmodified if we start with any nonminimal rational graph of a 3-manifold $M$ instead of $S^{3}$. If we wish to have natural well-defined surgery properties, it is convenient to consider only the case where $M$ is an integral homology sphere. On the other hand, this is rather restrictive for negative-definite rational graphs, as there are only two possibilities: $M=S^{3}$, treated above, and $M=\Sigma(2,3,5)$, the Poincaré 3-sphere oriented as the link of the surface singularity $\left\{x^{2}+y^{3}+z^{5}=0\right\}$. In particular, by identical proof we obtain the analogous theorem in this case.

Theorem 12 Consider any negative-definite nonminimal plumbing graph of $\Sigma=$ $\Sigma(2,3,5)$, put some arrows on the vertices, and regard them as link components of $K$ in $\Sigma$. Then the surgery manifold $\Sigma(K)$ for all sufficiently large surgery coefficients is an $L$-space.

Characterization of surgeries on rational homology spheres involves more technical data and identification (besides the surgery coefficients). Without doing these identifications we mention the following. If we drop above the integral homology restriction, we can start with any (not necessarily minimal) rational graph (this family is very large), and the algorithm still runs; nevertheless, in this way we have to consider only those surgeries that can be realized by the above construction (steps $\Gamma_{0} \mapsto \widetilde{\Gamma}_{0} \mapsto \Gamma^{\prime}$ ). For these surgeries, the statement and the proof still holds.

Acknowledgments The authors would like to thank M Hedden, J Hom, R Lipshitz, C Manolescu, P Ozsváth and Z Szabó for useful discussions. Special thanks to Jonathan Hanselman for sharing his program [6] (based on [7]), which helped us to formulate Theorem 7 and verify it in many examples. The first author is partially supported by the grant RFBR-13-01-00755 and NSF grant DMS-1559338. The second author is partially supported by OTKA Grant 100796. 


\section{References}

[1] M Artin, Some numerical criteria for contractability of curves on algebraic surfaces, Amer. J. Math. 84 (1962) 485-496 MR0146182

[2] M Artin, On isolated rational singularities of surfaces, Amer. J. Math. 88 (1966) 129-136 MR0199191

[3] E Gorsky, A Némethi, Lattice and Heegaard Floer homologies of algebraic links, Int. Math. Res. Not. 2015 (2015) 12737-12780 MR3431635

[4] E Gorsky, A Némethi, On the set of L-space surgeries for links, preprint (2015) arXiv: 1509.01170

[5] R L Graham, D E Knuth, O Patashnik, Concrete mathematics: a foundation for computer science, 2nd edition, Addison-Wesley, Reading, MA (1994) MR1397498

[6] J Hanselman, Computing $\widehat{\mathrm{HF}}$ of graph manifolds Python module Available at http://math.columbia.edu/ jhansel/graph_manifolds_program.html

[7] J Hanselman, Bordered Heegaard-Floer homology and graph manifolds, PhD thesis, Columbia University (2014) MR3232288 http://search.proquest.com/ docview/1528550664

[8] M Hedden, On knot Floer homology and cabling, II, Int. Math. Res. Not. 2009 (2009) 2248-2274 MR2511910

[9] J Hom, A note on cabling and L-space surgeries, Algebr. Geom. Topol. 11 (2011) 219-223 MR2764041

[10] T Kadokami, M Shimozawa, Dehn surgery along torus links, J. Knot Theory Ramifications 19 (2010) 489-502 MR2646642

[11] H B Laufer, On rational singularities, Amer. J. Math. 94 (1972) 597-608 MR0330500

[12] P Lisca, A I Stipsicz, Ozsváth-Szabó invariants and tight contact 3-manifolds, III, J. Symplectic Geom. 5 (2007) 357-384 MR2413308

[13] Y Liu, L-space surgeries on links (2013) arXiv:1409.0075 To appear in Quantum Topol.

[14] A Némethi, On the Ozsváth-Szabó invariant of negative definite plumbed 3-manifolds, Geom. Topol. 9 (2005) 991-1042 MR2140997

[15] W D Neumann, A calculus for plumbing applied to the topology of complex surface singularities and degenerating complex curves, Trans. Amer. Math. Soc. 268 (1981) 299-344 MR632532

[16] W D Neumann, F Raymond, Seifert manifolds, plumbing, $\mu$-invariant and orientation reversing maps, from: "Algebraic and geometric topology", (K C Millett, editor), Lecture Notes in Math. 664, Springer, Berlin (1978) 163-196 MR518415

[17] P Ozsváth, Z Szabó, Holomorphic disks and knot invariants, Adv. Math. 186 (2004) 58-116 MR2065507 
[18] P Ozsváth, Z Szabó, Holomorphic disks and topological invariants for closed threemanifolds, Ann. of Math. 159 (2004) 1027-1158 MR2113019

[19] P Ozsváth, Z Szabó, On knot Floer homology and lens space surgeries, Topology 44 (2005) 1281-1300 MR2168576

[20] P Ozsváth, Z Szabó, Holomorphic disks, link invariants and the multi-variable Alexander polynomial, Algebr. Geom. Topol. 8 (2008) 615-692 MR2443092

[21] M Spivakovsky, Sandwiched singularities and desingularization of surfaces by normalized Nash transformations, Ann. of Math. 131 (1990) 411-491 MR1053487

EG: Department of Mathematics, University of California, Davis

One Shields Avenue, Davis, CA 95616, United States

EG: International Laboratory of Representation Theory and Mathematical Physics

NRU-HSE, Vavilova 7, Moscow, Russia

AN: Algebraic Geometry and Differential Topology, Alfréd Rényi Institute of Mathematics Reáltanoda u. 13-15, Budapest, 1053, Hungary

egorskiy@math.ucdavis.edu, nemethi.andras@renyi.mta.hu

Received: 15 May 2014 Revised: 26 September 2015 